JOURNAL OF THE

CHUNGCHEONG MATHEMATICAL SOCIETY

Volume 26, No. 3, August 2013

\title{
A CENTRAL LIMIT THEOREM FOR LINEAR PROCESSES UNDER LINEAR NEGATIVELY QUADRANT DEPENDENCE
}

\author{
Hyun-Chull Kim*
}

\begin{abstract}
In this paper we establish a central limit theorem for weighted sums of $Y_{n}=\sum_{i=1}^{n} a_{n, i} X_{i}$, where $\left\{a_{n, i}, n \in N, 1 \leq i \leq n\right\}$ is an array of nonnegative numbers such that $\sup _{n \geq 1} \sum_{i=1}^{n} a_{n, i}^{2}<$ $\infty, \max _{1 \leq i \leq n} a_{n, i} \rightarrow 0$ and $\left\{X_{i}, i \in N\right\}$ is a sequence of linear negatively quadrant dependent random variables with $E X_{i}=0$ and $E X_{i}^{2}<\infty$. Using this result we will obtain a central limit theorem for partial sums of linear processes.
\end{abstract}

\section{Introduction}

For a sequence $\left\{a_{n}, n \geq 1\right\}$ of real numbers the limit superior is defined by $\inf _{r \geq 1} \sup _{n \geq r} a_{n}$ and is denoted by $\lim \sup _{n \rightarrow \infty} a_{n}$. Let $\left\{X_{n}, n \geq\right.$ $1\}$ be a sequence of random variables and $\left\{a_{n, k}, n \geq 1,1 \leq k \leq n\right\}$ be an array of real numbers. The weighted sums $\sum_{k=1}^{n} a_{n, k} X_{k}$ can play an important role in various applied and theoretical problems, such as those of the least squares estimators(see Kafles and Bhaskara Rao(1982)) and M-estimates(see Rao and Zhao(1992)) in linear models, the nonparametric regression estimators(see Priestley and Chao(1972)), etc. So the study of the central limit theorem is every important and significant.

Two random variables $X$ and $Y$ are said to be negatively quadrant dependent(NQD) [resp. positively quadrant dependent(PQD)] if $P(X \leq$ $x, Y \leq y)-P(X \leq x) P(Y \leq y) \leq 0$ [resp. $\geq 0$ ] for all $x, y \in R$.

A sequence $\left\{X_{k}, k \geq 1\right\}$ is said to be linear negatively[resp. positively] quadrant dependent(LNQD)[resp.(LPQD)] if for any disjoint finite subsets $A, B \subset N$ and any positive real numbers $r_{j}, \sum_{i \in A} r_{i} X_{i}$

Received June 12, 2013; Accepted July 19, 2013.

2010 Mathematics Subject Classification: Primary 60F05.

Key words and phrases: linear processes, linear negatively quadrant dependent, central limit theorem, weighted sum, uniformly integrable.

*This paper was supported by the Sehan University Research Fund in 2013. 
and $\sum_{j \in B} r_{j} X_{j}$ are NQD[resp. PQD]. The definition of NQD is given by Lehmann(1966) and the concept of LNQD[resp. LPQD] is given by Newman(1984). Because of their wide applications Birkel(1993) gave a central limit theorem and a functional central limit theorem for LPQD sequence.

Theorem 1.1 (Newman(1984)). Let $\left\{X_{n}, n \geq 1\right\}$ be a sequence of strictly stationary $L N Q D$ random variables with $E X_{1}=0$ and $E X_{1}^{2}<$ $\infty$. If

$$
\sigma^{2}=\sum_{i=1}^{\infty} \operatorname{Cov}\left(X_{1}, X_{i}\right)<\infty
$$

then

$$
\sigma^{-1} n^{-\frac{1}{2}} \sum_{i=1}^{n} X_{i} \rightarrow^{D} N(0,1) \text { as } n \rightarrow \infty,
$$

where $\rightarrow^{D}$ means convergence in distribution.

Peligrad and Utev(1997) have proved the following central limit theorem for weighted sums of associated random variables :

Theorem 1.2. Let $\left\{a_{n, i}, 1 \leq i \leq n, n \geq 1\right\}$ be an array of nonnegative numbers such that

$$
\begin{gathered}
\sup _{n \geq 1} \sum_{i=1}^{n} a_{n, i}^{2}<\infty, \\
\max _{1 \leq i \leq n} a_{n, i} \rightarrow 0 \text { as } n \rightarrow \infty .
\end{gathered}
$$

Let $\left\{X_{n}, n \geq 1\right\}$ be a sequence of mean zero associated random variables such that

$$
\left\{X_{i}^{2}\right\} \text { is an uniformly integrable family, }
$$

and

$$
\sum_{j:|k-j| \geq u} \operatorname{Cov}\left(X_{k}, X_{j}\right) \rightarrow 0 \text { as } u \rightarrow \infty \text { uniformly in } k \geq 1 .
$$

(See Cox and Grimmett(1984)). Then

$$
\sum_{i=1}^{n} a_{n, i} X_{i} \rightarrow^{D} N(0,1) \text { as } n \rightarrow \infty,
$$

where $\rightarrow^{D}$ means convergence in distribution. 
In this paper inspired by Peligrad and Utev(1997) we extend Theorem $B$ to the case of LNQD random variables and prove the central limit theorem for linear process generated by LNQD random variables using this result.

\section{Main results}

TheOREM 2.1. Let $\left\{X_{n}, n \geq 1\right\}$ be a sequence of mean zero and finite variance $L N Q D$ random variables satisfying (1.3), (1.4) and

$$
\sum_{j:|k-j| \geq u}\left|\operatorname{Cov}\left(X_{k}, X_{j}\right)\right| \rightarrow 0 \text { as } u \rightarrow \infty \text { uniformly in } k \geq 1 \text {. }
$$

Let $\left\{a_{n, i}, 1 \leq i \leq n, n \geq 1\right\}$ be an array of nonnegative numbers satisfying (1.1) and (1.2).

Then

$$
\sum_{i=1}^{n} a_{n, i} X_{i} \rightarrow^{D} N(0,1) \text { as } n \rightarrow \infty .
$$

The following lemma needs to prove Theorem 2.1.

Lemma 2.2 (Newman(1984)). Let $\left\{X_{n}, n \geq 1\right\}$ be a sequence of $L N Q D$ random variables with finite second moments. Then

$$
\left|E \exp \left(i t \sum_{j=1}^{n} X_{j}\right)-\prod_{j=1}^{n} E\left(\exp i t X_{j}\right)\right| \leq C t^{2}\left|\operatorname{Var}\left(\sum_{j=1}^{n} X_{j}\right)-\sum_{j=1}^{n} \operatorname{Var}\left(X_{j}\right)\right|
$$

for all $t \in R$, where $C>0$ is an arbitrary constant, not depending on $n$.

REMARK 2.3. The right hand side of (2.2) converges to zero as $n$ goes infinity.

Proof of Theorem 2.1. Without loss of generality we assume that $a_{n, i}=$ 0 for $i>n$. For $1 \leq a<b \leq n$ and $1 \leq u \leq b-a$ we have, after simple manipulations

$$
\begin{aligned}
(2.3) 0 & \leq \sum_{i=a}^{b-u} a_{n, i} \sum_{j=i+u}^{b} a_{n, j}\left|\operatorname{Cov}\left(X_{i}, X_{j}\right)\right| \\
& \leq \sup _{k}\left(\sum_{j:|k-j| \geq u}\left|\operatorname{Cov}\left(X_{k}, X_{j}\right)\right|\right)\left(\sum_{i=a}^{b} a_{n, i}^{2}\right) .
\end{aligned}
$$


In particular, by $(1.5)^{\prime}$ there exists a constant $C$ such that for every $1 \leq a \leq b \leq n$,

$$
\operatorname{Var}\left(\sum_{i=a}^{b} a_{n, i} X_{i}\right) \leq C \sum_{i=a}^{b} a_{n, i}^{2} .
$$

We shall construct an array of random variables $\left\{Z_{n, i}, 1 \leq i \leq n, n \geq 1\right\}$ for which we shall make use of Lemma 2.2. Fix a small positive $\epsilon$ and find a positive $u=u_{\epsilon}$ such that

$$
\begin{aligned}
0 & \leq \sum_{i=1}^{b-u} a_{n, i} \sum_{j=i+u}^{b} a_{n, j}\left|\operatorname{Cov}\left(X_{i}, X_{j}\right)\right| \\
& \leq \epsilon \text { for every } n \geq u+1 .
\end{aligned}
$$

This is possible because of (2.3) and (1.5)'.

As in Peligrad and Utev(1997) we denote $[x]$ the integer part of $x$ and define

$$
\begin{aligned}
K & =\left[\frac{1}{\epsilon}\right] \\
Y_{n, j} & =\sum_{i=u j+1}^{u(j+1)} a_{n, i} X_{i}, j=0,1, \cdots \\
A_{j} & =\left\{i: 2 K j \leq i<2 K j+K,\left|\operatorname{Cov}\left(Y_{n, i}, Y_{n, i+1}\right)\right|\right. \\
& \left.\leq \frac{2}{K} \sum_{i=2 K j}^{2 K j+K} \operatorname{Var}\left(Y_{n, i}\right)\right\} .
\end{aligned}
$$

From the fact that $2\left|\operatorname{Cov}\left(Y_{n, i}, Y_{n, i+1}\right)\right| \leq \operatorname{Var}\left(Y_{n, i}\right)+\operatorname{Var}\left(Y_{n, i+1}\right)$ we get that for every $j$ the set $A_{j}$ is not empty.

Now we define the integers $m_{1}, m_{2}, \cdots, m_{n}$ recursively by $m_{0}=0$. $m_{j+1}=\min \left\{m: m>m_{j}, m \in A_{j}\right\}$ and define

$$
\begin{aligned}
Z_{n, j} & =\sum_{i=m_{j}+1}^{m_{j+1}} Y_{n, i}, j=0,1, \cdots \\
\Delta_{j} & =\left\{u\left(m_{j}+1\right)+1, \cdots, u\left(m_{j+1}+1\right)\right\} .
\end{aligned}
$$

We observe that

$$
Z_{n, j}=\sum_{k \in \Delta_{j}} a_{n, k} X_{k}, j=0,1, \cdots .
$$

(See Peligrad and Utev(1997).)

It is easy to see that every set $\Delta_{j}$ contains no more that $3 K u$ elements. 
Hence, for every fixed positive by (1.1) and (1.2) the array $\left\{Z_{n, i}: i=\right.$ $1, \cdots, n, n \geq 1\}$ satisfies the Lindeberg's condition. It remains to see that by Lemma 2.2

$$
\begin{aligned}
& \left|E \exp \left(i t \sum_{j=1}^{n} Z_{n, j}\right)-\Pi_{j=1}^{n} \operatorname{Eexp}\left(i t Z_{n, j}\right)\right| \\
& \leq C t^{2}\left(\operatorname{Var}\left(\sum_{j=1}^{n} Z_{n, j}\right)-\sum_{j=1}^{n} \operatorname{Var}\left(Z_{n, j}\right)\right) \\
& \leq C t^{2}\left(2 \sum_{i=1}^{n}\left|\operatorname{Cov}\left(Z_{n, i}, Z_{n, i+1}\right)\right|+2 \sum_{i=1}^{n-2} \sum_{j=i+2}^{n}\left(\left|\operatorname{Cov}\left(Z_{n, i}, Z_{n, j}\right)\right|\right)\right) \\
& \leq C t^{2}\left\{4 \sum_{i=1}^{n-u} a_{n, i} \sum_{j=i+u}^{n} a_{\mathbf{n}, \mathbf{j}}\left|\operatorname{Cov}\left(X_{i}, X_{j}\right)\right|+2 \sum_{j=1}^{n}\left|\operatorname{Cov}\left(Y_{n, m_{j}}, Y_{n, m_{j}+1}\right)\right|\right\} \\
& \leq C t^{2}\left\{4 \epsilon+\frac{8}{K} \sum_{i=1}^{n} \operatorname{Var}\left(Y_{n, i}\right)\right\} \\
& \leq C^{\prime} t^{2} \epsilon\left(1+\operatorname{Var}\left(\sum_{i=1}^{n} a_{n, i} X_{i}\right)\right) \\
& \leq C^{\prime \prime} t^{2} \epsilon \text { for every positive } \epsilon .
\end{aligned}
$$

Finally, the proof Theorem 2.1 is complete by Theorem 4.2 in Billingsley(1968).

Corollary 2.4. $\left\{X_{i}, i \geq 1\right\}$ be a sequence of mean zero LNQD random variables such that $\left\{X_{i}^{2}\right\}$ is uniformly integrable and $\left\{a_{n, i}^{\prime}, 1 \leq\right.$ $i \leq n, n \geq 1\}$ be an array of nonnegative numbers such that

$$
\sup _{n \geq 1} \sum_{i=1}^{n} \frac{a_{n, i}^{\prime}}{\sigma_{n}^{2}}<\infty
$$

$$
\max _{1 \leq i \leq n} \frac{a_{n, i}^{\prime}}{\sigma_{n}^{2}} \rightarrow 0 \text { as } n \rightarrow \infty,
$$

where $\sigma_{n}^{2}=\operatorname{Var}\left(\sum_{i=1}^{n} a_{n, i}^{\prime} X_{i}\right)$.

If $(1.5)^{\prime}$ holds, then

$$
\frac{1}{\sigma_{n}} \sum_{i=1}^{n} a_{n, i}^{\prime} X_{i} \rightarrow^{D} N(0,1) \text { as } n \rightarrow \infty .
$$


Proof. Let $a_{n, i}=\frac{a_{n, i}^{\prime}}{\sigma_{n}}$. Then by Theorem 2.1 the result (2.6) follows.

In time series analysis, the linear process $Y_{m}=\sum_{j=1}^{\infty} a_{m+j} X_{j}$ is of great importance, where $\left\{a_{k}, 0<k<\infty\right\}$ is a sequence of numbers and many important time series models have the type of $Y_{m}$.

We apply Theorem 2.1 to obtain the following central limit theorem for the partial sum of a linear process of the form $Y_{m}=\sum_{j=1}^{\infty} a_{m+j} X_{j}$.

Theorem 2.5. $Y_{m}=\sum_{j=1}^{\infty} a_{m+j} X_{j}$, where $\left\{a_{k}, 0<k<\infty\right\}$ is a sequence of nonnegative numbers with $\sum_{j} a_{j}<\infty$ and $\left\{X_{j}\right\}$ is a sequence of $L N Q D$ random variables satisfying (1.4) and (1.5)'. Set

$$
\operatorname{Var}\left(S_{n}^{*}\right)=\sigma_{n}^{* 2}
$$

where $S_{n}^{*}=\sum_{m=1}^{n} Y_{m}$. If $\sigma_{n}^{*} \rightarrow \infty$, then

$$
\frac{S_{n}^{*}}{\sigma_{n}^{*}} \rightarrow^{D} N(0,1)
$$

where $\rightarrow^{D}$ means convergence in distribution.

Proof. The proof is similar to that of Theorem 2.4 in Peligrad and Utev(1997). To complete the proof we repeat it here. Without loss of generality we assume

$$
\sup _{n \geq 1} E\left(X_{n}^{2}\right)=1
$$

We also have

$$
S_{n}^{*}=\sum_{m=1}^{n} Y_{m}=\sum_{j=1}^{\infty}\left(\sum_{m=1}^{n} a_{m+j}\right) X_{j}
$$

In order to apply Theorem 2.1, we choose $W_{n}$ such that $\sum_{j>W_{n}} a_{j}^{2}<n^{-3}$ and take $k_{n}=W_{n}+n$. Then

$$
\frac{S_{n}^{*}}{\sigma_{n}^{*}}=\sum_{j=1}^{k_{n}}\left(\sum_{k=1}^{n} a_{k+j}\right) X_{j} / \sigma_{n}^{*}+\sum_{j=k_{n}+1}^{\infty}\left(\sum_{k=1}^{n} a_{k+j}\right) X_{j} / \sigma_{n}^{*}=T_{n}+U_{n}
$$


By the Cauchy-Schwarz inequality and the assumption we have the following estimate

$$
\begin{aligned}
\operatorname{Var}\left(U_{n}\right) & \left.\leq \sum_{j=k_{n}+1}^{\infty}\left(\sum_{k=1}^{n} a_{k+j}\right) X_{j} / \sigma_{n}^{*}\right)^{2} \\
& \leq n\left(\sigma_{n}^{*}\right)^{-2} \sum_{j=k_{n}+1}^{\infty} \sum_{k=1}^{n} a_{k+j}^{2} \\
& \leq n^{2}\left(\sigma_{n}^{*}\right)^{-2} \sum_{j=k_{n}-n+1}^{\infty} a_{j}^{2} \\
& \leq n^{2}\left(\sigma_{n}^{*}\right)^{-2} \sum_{j=W_{n}+1}^{\infty} a_{j}^{2} \\
& \leq n^{-1}\left(\sigma_{n}^{*}\right)^{-2} \rightarrow 0 \text { as } n \rightarrow \infty,
\end{aligned}
$$

which yields

$$
U_{n} \rightarrow 0 \text { in probability as } n \rightarrow \infty .
$$

By Theorem 4.1 of Billingsley(1968) it remains only to prove that $T_{n} \rightarrow^{D}$ $N(0,1)$ as $n \rightarrow \infty$.

Put

$$
a_{n, k}=\frac{\sum_{j=1}^{n} a_{k+j}}{\sigma_{n}^{*}} .
$$

From the assumptions $\sum_{j} a_{j}<\infty, \sigma_{n}^{*} \rightarrow \infty$ and (2.9) we obtain

$$
\sup _{k \geq 1} \sum_{j=1}^{n} a_{k+j} / \sigma_{n}^{*} \rightarrow 0 \text { as } n \rightarrow \infty,
$$

which yields

$$
\max _{1 \leq k \leq n} a_{n, k} \rightarrow 0 \text { as } n \rightarrow \infty .
$$

In order to apply Theorem 2.1 we have to show

$$
\sup _{n \geq 1} \sum_{k=1}^{n} a_{n, k}^{2}<\infty .
$$

Suppose on the contrary that for some $\epsilon>0$ there exists a subsequence $\left(j^{\prime}, n^{\prime}\right), n^{\prime} \rightarrow \infty$ such that

$$
\sum_{k=1}^{n} a_{k+j^{\prime}}>\epsilon \sigma_{n}^{*}
$$


Denote by $A=\sup _{k \geq 1} a_{k}$ and notice that for $r>j^{\prime}$,

$$
\sum_{k=1}^{n} a_{k+r} \geq \epsilon \sigma_{n^{\prime}}-2 A\left(r-j^{\prime}\right) .
$$

Hence

$$
\begin{aligned}
\frac{\sigma_{n^{\prime}}^{2}}{b} & \geq \sum_{i=j^{\prime}}^{j+W}\left(\sum_{k=1}^{n} a_{k+j}\right)^{2} \\
& \geq W \epsilon^{2} \sigma_{n^{\prime}}^{2}-4 A \sigma_{n^{\prime}} \epsilon\left(\sum_{i=j^{\prime}}^{j+W}\left(i-j^{\prime}\right)\right) \\
& \geq W \epsilon^{2} \sigma_{n^{\prime}}^{2}-4 A \sigma_{n^{\prime}} \epsilon W^{2} .
\end{aligned}
$$

Taking $W$ to be the least positive integer greater than of equal to $\frac{3}{b \epsilon^{2}}$ and because $\sigma_{n^{\prime}} \rightarrow \infty$, we obtain for $n^{\prime}$ sufficiently large,

$$
\frac{\sigma_{n^{\prime}}^{2}}{b} \geq \frac{3 \sigma_{n^{\prime}}^{2}}{b}-\sigma_{n^{\prime}} \frac{3 \sigma A}{b^{2} \epsilon^{2}}>\frac{2 \sigma_{n^{\prime}}^{2}}{b}
$$

which is a contradiction. That is we have

$$
\sup _{n \geq 1} \sum_{k=1}^{n} a_{n, k}^{2}<\infty
$$

Hence, by (2.10) and (2.11) we have

$$
T_{n} \rightarrow^{D} N(0,1) .
$$

Finally, by (2.8), (2.12) and Theorem 4.1 of Billingsley the result follows.

\section{References}

[1] P. Billingsley, Convergence of Probability Measures, Wiley, New York, 1968.

[2] T. Birkel, A functional central limit theorem for positively dependent random variables, J. Multivariate Anal. 11 (1993), 314-320.

[3] J. T. Cox and G. Grimmett, Central limit theorems for associated random variables and the percolation model, Ann. Probab. 12 (1984), 514-528.

[4] D. Kafles and R. M. Bhaskara, Weak consistency of least squares estimators in linear models, J. Multivariate Anal. 12 (1982), 186-198.

[5] E. L. Lehmann, Some concepts of dependence, Ann. Math. Stat. 37 (1966), $1137-1153$

[6] C. M. Newman, Normal fluctuations and the FKG inequalities, Commun. Math. Phys. 74 (1980), 119-128. 
[7] C. M. Newman, Asymptotic independence and limit theorems for positively and negatively dependent random variables, in Inequalities in Statistics and Probability (Tong, Y.L., ed., IMS, Hayward, CA), 1984.

[8] M. Peligrad and S. Utev, Central limit theorem for linear processes, Ann. Probab. 25 (1997), 443-456.

[9] M. B. Priestley and M. T. Chao, Nonparametric function fitting, J. Roy. Statist. Soc. Ser. B 34 (1972), 385-392.

[10] C. R. Rao and M. T. Zhao, Linear representation of M-estimates in linear models, Canad. J. Statist. 20 (1992), 359-368.

[11] G. Roussas, Asymptotic normality of random fields of positively or negatively associated processes, J. Multivariate Anal. 50 (1994), 152-173.

[12] S. Shanskin, A strong invariance principle for postively or negatively associated random fields, Statist. Probab. Lett. 78 (2008), 2121-2129.

*

Department of Mathematics Education

Sehan University

Jeonnam 526-720, Korea

E-mail: kimhc@sehan.ac.kr 